\title{
Rapid Compositional EELS and EDS Mapping of Amorphous Silicate Matrix in Extraterrestrial Materials
}

Kenta Ohtaki ${ }^{1}$, John Bradley ${ }^{2}$, Hope Ishii ${ }^{2}$, Jim Ciston $^{3}$ and Karen Bustillo ${ }^{4}$

${ }^{1}$ University of California-Irvine, Irvine, California, United States, ${ }^{2}$ University of Hawaii at Manoa, Honolulu, Hawaii, United States, ${ }^{3}$ Lawrence Berkeley National Laboratory, Berkeley, California, United States, ${ }^{4}$ NCEM, Molecular Foundry, LBNL, Berkeley, California, United States

Materials from cosmically primitive objects like comets and the least-altered asteroids best represent the ancient starting materials from the formation of our Solar System. Energy dispersive x-ray spectroscopy (EDS) elemental mapping with 2-5 nanometer-scale spatial resolution has been used to compare amorphous silicates and organic carbon in cometary dust from chondritic porous interplanetary dust particles (CP IDPs) and in fine-grained matrix from primitive meteorites from asteroids [1]. Comet dust has highly reduced chemistry in contrast to asteroid materials that have undergone varying degrees of oxidation. Thus, obtaining oxidation state maps on the same scale as the elemental maps can provide a new means of exploring the relationship between comet and asteroid materials. Oxidation states can be measured and mapped using electron energy-loss spectroscopy (EELS) and energy-filtered imaging, but conventional EEL spectrometers have low signal-to-noise $(\mathrm{S} / \mathrm{N})$ and limited energy range $(1000 \mathrm{eV})$.

A new generation electron energy-loss spectrometer, the Continuum (Gatan, Inc.), uses the K3 ${ }^{\mathrm{TM}}$ single electron-counting CMOS detector to collect high-resolution EELS maps 40 times faster than a conventional scintillator camera. The Continuum's electron counting mode detects the centroid of electron strikes above the noise floor and almost entirely removes the fixed-pattern readout noise of conventional detectors. Operation in this mode greatly increases $\mathrm{S} / \mathrm{N}$ for low intensity signals and minimizes electron beam damage. The modulation transfer function of the detector also reduces bloom between pixels and allows for more efficient use of energy channels at lower spectrometer dispersions. The high $\mathrm{S} / \mathrm{N}$ enables rapid mapping of oxidation states at $2-5 \mathrm{~nm}$ spatial scales. With a $3000 \mathrm{eV}$-wide energy range, the zero loss peak and core loss spectra are recorded simultaneously, eliminating energy drift issues and enabling on-the-fly deconvolution of plural scattering effects.

This study focuses on the formation mechanism of the amorphous silicates found in primitive meteorite matrix, inferred from their compositions and oxidation states. Amorphous silicates are expected to carry information on processes experienced before/during accretion and on their parent bodies. Meteorites Paris and Acfer 094 were chosen for this work because they are believed to have experienced minimal alteration by parent body processing, and both contain amorphous silicate-rich "GEMS-like" material similar to that in CP IDPs [2,3]. TEM specimens were extracted from metal-rich regions, using conventional FIB trenching and a hybrid ultramicrotomy-FIB method [4], and mapped with EDS and EELS. Fe oxidation state distributions, chemical shifts, and core-loss fine-structures were measured using EELS ( 100 meV FWHM ZLP) over 0-3000 eV using the Continuum spectrometer.

EELS and EDS spectral maps were collected from multiple locations in Paris and Acfer 094, but this report focuses on a single representative location in Acfer 094 with amorphous silicate and forsterite $\left(\mathrm{Mg}_{2} \mathrm{SiO}_{4}\right)$ crystals. The forsterite crystals Fo 1 and Fo 2 shown in Fig. 1 have different morphologies; Fo 1 has "fuzzy" crystal edges whereas Fo 2 has more distinct edges. "Fuzzy" edges are typical of partly altered crystals and suggest that Fo 1 is partly altered from crystalline to an amorphous phase. The Fe oxidation state map using $\mathrm{Fe}-\mathrm{L}$ shows that $\mathrm{Fe}^{3+}$ resides in the amorphous silicate matrix and $\mathrm{Fe}^{2+}$ is in the 
Fe-sulfide grains. The higher oxidation state in the matrix suggests that the meteorite experienced an oxidizing environment. The amorphous silicate matrix has an $\mathrm{O}-\mathrm{K}$ pre-edge consistent with the presence of hydroxyl (-OH) [5], and others report high water content [6], so the phase change is likely due to aqueous alteration. Peak splitting of the O-K edge due to molecular orbital $t_{2 g}-e_{g}$ splitting [7] is more pronounced in the (less-altered) Fo 2 crystal (Fig. 1d) than Fo 1 (Fig. 1a), and the O-K splitting is almost absent in the Mg-rich nano-crystalline silicate (nc-Fo) (Fig. 1b) and completely absent in the amorphous silicate matrix (Fig. 1c). Based on its electron diffraction pattern and lack of O-K edge splitting, the ncFo phase has lost long range crystal integrity. This suggests that the nc-Fo phase formed from larger single crystal forsterite grain(s) via alteration.

EDS shows that nc-Fo has less Mg and more Fe than the crystalline Fo 1 and 2 grains (Table 1). Depletion of $\mathrm{Mg}$ due to aqueous alteration has been reported previously and supports the role of aqueous alteration in Acfer 094 amorphous silicate formation [8]. In other locations where Fe-sulfide grains are embedded in amorphous silicate matrix, $\mathrm{Fe}^{(3+)}$ concentration declines outwards into the silicate matrix. (Lower Fe concentration is also observed in amorphous silicate next to (Fe-free) forsterite.) Hopp et al. [9] report a similar trend in oxidation state in Acfer 094 and similarly conclude that it is likely due to parent body aqueous alteration.

The formation mechanism(s) of amorphous silicates in chondritic materials is debated. Some propose direct condensation in the solar nebula prior to accretion [10,11], but our EELS oxidation maps combined with EDS and diffraction data in this study suggest an alternative formation route, amorphization of crystalline silicates (e.g. Fe-poor forsterite and pyroxene in Type I chondrules and Fe-rich Type II chondrules) and $\mathrm{Fe}^{3+}$ infiltration of the amorphous silicate from oxidative decomposition of Fe-metal and $\mathrm{Fe}^{2+}$-rich sulfides during parent body aqueous alteration.

Acknowledgements: This research is funded by NASA grant NNX16AK41G to HAI. Work at the Molecular Foundry was supported by the Office of Science, BES, U.S. DOE under Contract No. DEAC02-05CH11231 and MAS Goldstein Scholar Award

Table 1 EDS elemental composition ratios (at. \% ratios) in Fo 1, 2 and nc-Fo

\begin{tabular}{lccc}
\hline & $\mathrm{Mg} /(\mathrm{Mg}+\mathrm{Si}+\mathrm{Fe})$ & $\mathrm{Fe} /(\mathrm{Mg}+\mathrm{Si}+\mathrm{Fe})$ & $\mathrm{Si} /(\mathrm{Mg}+\mathrm{Si}+\mathrm{Fe})$ \\
\hline Fo 1 & 59 & 5.6 & 36 \\
nc-Fo & 48 & 19 & 34 \\
Fo 2 & 64 & 0.4 & 35 \\
\hline
\end{tabular}




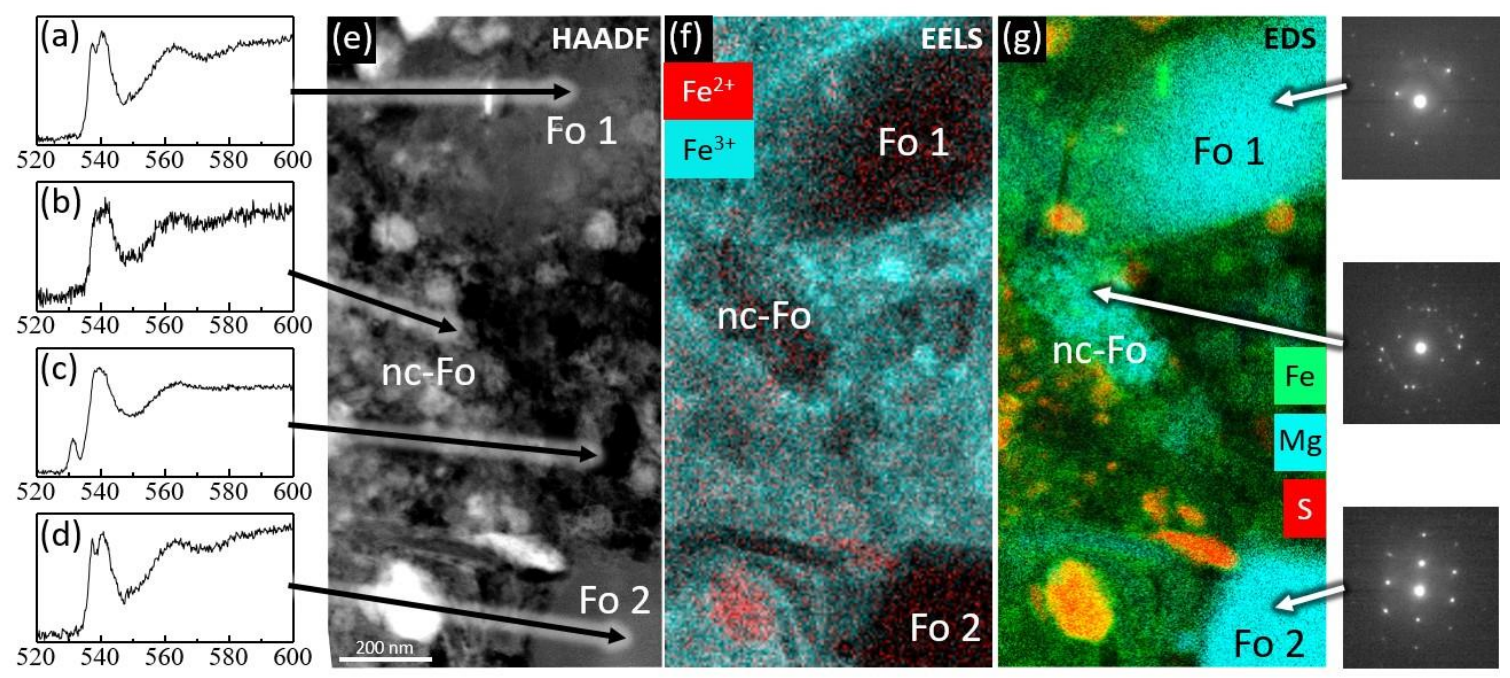

Figure 1. EELS O-K spectra (a) from the forsterite 1 (Fo1) crystal with "fuzzy" edges (b) nano-crystalline Mg-silicate (nc-Fo) (c) amorphous silicate matrix (d) forsterite 2 (Fo 2) crystal with defined edges and FeL EELS (f) and EDS (g) maps with (e) HAADF image. Electron nano-diffraction patterns show single crystal and nano-polycrystalline diffraction patterns.

\section{References}

[1] Ishii H. A. et al. MetSoc (2019) No. 2157, id.6405

[2] Leroux H. et al. GCA, 170 (2015) 247-265

[3] Vollmer C. et al. ApJ, 700 (2009) 774-782

[4] Ohtaki K. K. et al. (2019) Microsc. Microanal., in press (doi:10.1017/S1431927619015186)

[5] R. Wirth, Phys Chem Minerals 24 (1997) 561-568]

[6] Hewins R. et al. MetSoc (2019) No 2157, id. 6267

[7] C. C. Ahn, second edition Wiley-VCH (2004) 112

[8] A. Blum et al. Nature 331 (1988) 431-433

[9] Hopp et al. Meteoritics \& Planetary Science53, Nr 2, (2018) 153-166

[10] Brearley A. J. et al. GCA, 59 (1995) 4307-4316

[11] Abreu N. M. and Brearley A. J. GCA, 74 (2010) 1146-1171 